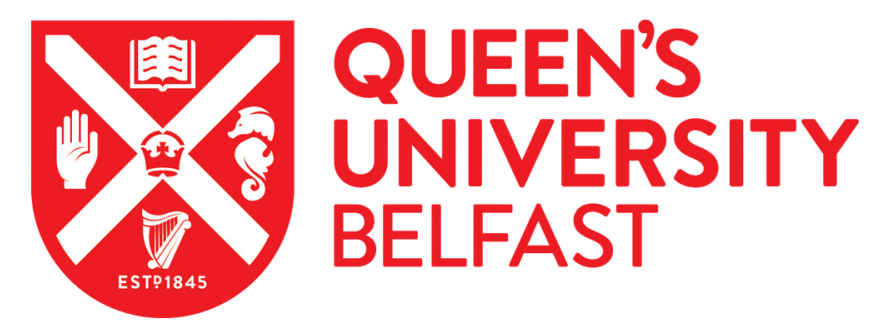

\title{
Preoperative characteristics and compliance with follow-up after trabeculectomy surgery in rural southern China
}

Jin, L., Li, L., Zheng, S., Dan, A., Chen, T., Wang, X., Li, G., \& Congdon, N. (2017). Preoperative characteristics and compliance with follow-up after trabeculectomy surgery in rural southern China. British Journal of Ophthalmology, 101(2). https://doi.org/10.1136/bjophthalmol-2015-308331

Published in:

British Journal of Ophthalmology

Queen's University Belfast - Research Portal:

Link to publication record in Queen's University Belfast Research Portal

Publisher rights

(c) 2016 The Authors.

This article has been accepted for publication in

British Journal of Opthalmology following peer

review. The definitive copyedited, typeset version

Jin, L, Li, L, Zheng, S, Dan, A, Chen, T, Wang, X, Li, G \& Congdon, N 2016, 'Preoperative characteristics and compliance with follow-up after trabeculectomy surgery in rural southern China' British journal of ophthalmology is available online at: www.

http://bjo.bmj.com/content/101/2/131

General rights

Copyright for the publications made accessible via the Queen's University Belfast Research Portal is retained by the author(s) and / or other copyright owners and it is a condition of accessing these publications that users recognise and abide by the legal requirements associated with these rights.

Take down policy

The Research Portal is Queen's institutional repository that provides access to Queen's research output. Every effort has been made to ensure that content in the Research Portal does not infringe any person's rights, or applicable UK laws. If you discover content in the Research Portal that you believe breaches copyright or violates any law, please contact openaccess@qub.ac.uk. 


\section{Pre-Operative Characteristics and Compliance with Follow-up after}

Trabeculectomy Surgery in Rural Southern China

Ke Yang ${ }^{1,2}$, Ling $\mathrm{Jin}^{1}, \mathrm{Li} \mathrm{Li}^{2}$,Siming Zeng ${ }^{2}$,Aihua $\mathrm{Dan}^{3}$,Tingting Chen ${ }^{1}$,Xiuqin wang ${ }^{1,4}$,Guirong $\mathrm{Li}^{2}$,Nathan Congdon $\mathrm{MD}^{1,5,6}$

${ }^{1}$ State Key Laboratory of Ophthalmology, Zhongshan Ophthalmic Center, Sun Yat-sen University, Guangzhou, China

${ }^{2}$ Department of Ophthalmology, People's Hospital of Guangxi Zhuang Autonomous Region, Nanning, China

${ }^{3}$ Red Cross Hospital of Yunnan Province, Kunming, China

${ }^{4}$ Affiliated Hospital of Guangdong Medical College, Zhanjiang, Guangdong, China ${ }^{5}$ ORBIS international, New York, USA

${ }^{6}$ Queen's University Belfast, Belfast, Northern Ireland

Address for Correspondence: Prof Nathan Congdon, State Key Laboratory of Ophthalmology, Zhongshan Ophthalmic Center, Guangzhou China 510060, or TREE Centre, Centre for Public Health, Queen's University Belfast, Belfast UK BT12 6AB; ncongdon1@gmail.com

Key words: Glaucoma, trabeculectomy, compliance, follow-up, rural, China

Manuscript length: Text: 3282 words, 5 tables, Abstract: 245 words

Draft: 1 March 2016 
Subtitle: The beneficial impact of glaucoma surgery in rural China is limited by poor post-operative follow-up and delays in accessing care, with significant vision loss already present in the majority of patients. 


\begin{abstract}
PURPOSE To evaluate pre-operative characteristics and follow-up in rural China after trabeculectomy, the primary treatment for glaucoma there.
\end{abstract}

METHODS Patients undergoing trabeculectomy at 14 rural hospitals in Guangdong and Guangxi Provinces and their doctors completed questionnaires concerning clinical and sociodemographic information, transportation, and knowledge and attitudes about glaucoma. Follow-up after surgery was assessed as cumulative score (1 week: 10 points, 2 weeks: 7 points, one month: 5 points).

RESULTS Among 212 eligible patients, mean pre-operative presenting acuity in the operative eye was 6/120, with $61.3 \%(n=130)$ blind $(<=6 / 60)$. Follow-up rates were 60.8\% (129/212), 75.9\% (161/212) and 26.9\% (57/212) at 1 week, 2 weeks and 1 month. Patient predictors of poor follow-up included elementary education or less (OR=0.37, 95\% CI: 0.20, 0.70, $\mathrm{P}=0.002$ ), believing follow-up was not important (OR=0.62, 95\% CI: 0.41, 0.94, $\mathrm{P}=0.02)$, lack of an accompanying person $(\mathrm{OR}=0.14$, 95\% CI: 0.07, 0.29, P<0.001), family annual income $<$ USD800 (OR=0.28, 95\% CI: $0.11,0.72, \mathrm{P}=0.008$ ), and not requiring removal of scleral flap sutures post-operatively (OR=0.11, 95\% CI: 0.06, 0.22, $\mathrm{P}<0.001)$. Age, sex, employment, travel distance/time/costs, patient pre-operative clinical factors and physician factors were unassociated with follow-up.

CONCLUSIONS Follow-up after two weeks was inadequate to provide optimal clinical care, and surgery is being applied too late to avoid blindness in the majority of patients. Earlier surgery, support for return visits and better explanations of the importance of follow-up are needed. Directing all patients to return for possible scleral 
flap suture removal may be a valid strategy to improve follow up. 


\section{INTRODUCTION}

Glaucoma is the leading global cause of irreversible blindness. The number of people with glaucoma worldwide will increase from 64.3 million in 2013 to 111.8 million in 2040, disproportionally affecting people residing in Asia andAfrica. ${ }^{1}$ China is no exception, with $7.7-11 \%$ of blindness attributable to glaucoma. ${ }^{2-4}$ Medical and surgical therapies exist for glaucoma, and are trial-proven to reduce risk of disease progression if applied sufficiently early in the course. ${ }^{5-7}$ Without timely and successful treatment, blindness from glaucoma, when it occurs, is generally not reversible.

Though medical treatment for glaucoma remains a common first-line option in developed areas, lack of availability and high cost of medications make this modality less practical ${ }^{8}$ in areas of limited resources. Though few studies have been done, ${ }^{9}$ adherence with medical therapy for glaucoma also appears to be poor in developing areas. For these reasons, glaucoma surgery, such as trabeculectomy, may be preferred ${ }^{9,}$ ${ }^{10}$ as initial treatment for glaucoma in rural areas of lower and middle income countries. Early postoperative follow-up after trabeculectomy surgery is crucial to monitor treatment efficacy and may reduce the risk of visually-significant complications and surgical failure. Relatively non-invasive interventions such as removal of scleral flap sutures and bleb massage must generally be utilized in the early post-operative period ${ }^{11 \text {, }}$

12 in order to achieve successful control of intraocular pressure. Use of topical ocular steroid medications has also been demonstrated to improve outcomes of trabeculectomy surgery, ${ }^{13}$ and patients not adhering to recommended post-operative follow-up regimens may lose the opportunity both to replenish supplies of medication and to receive instruction and encouragement from providers on their use. Relatively 
little is known about rates of follow-up after glaucoma surgery in rural areas of low and middle income countries. A 2011 study of follow-up after trabeculectomy in China found that $84 \%$ of patients returned at 1 month after surgery, ${ }^{14}$ while a report from India involving urban and rural patients ${ }^{9}$ gave a figure of $58 \%$ at one year after trabeculectomy surgery in the setting of a randomized trial.

In order to find effective interventions to promote follow-up after glaucoma surgery in rural China, we carried out a prospective, clinic-based study to determine patient compliance with a standard regimen of clinic visits in the first month after trabeculectomy surgery, as well as patient and physician determinants of poor follow-up. The purpose of the study was to assess follow-up and its determinants. The study was also designed to assess patient pre-operative clinical characteristics, to determine whether treatment was being provided sufficiently early to reduce the burden of glaucoma blindness.

\section{METHODS}

The protocol for this study was approved in full by the Ethics Committee of the Zhongshan Ophthalmic Center, Sun Yat-sen University (Guangzhou, China). Oral informed consent was obtained from all subjects, and the tenets of the Declaration of Helsinki were followed throughout.

Consecutive eligible glaucoma patients undergoing trabeculectomy were enrolled at 14 rural county-level hospitals (7 in Guangdong Province and 7 in neighboring Guangxi, both in southern China) from January to December 2014. Guangdong's per capita Gross Domestic Product (GDP) of USD 9452 in 2013 was ranked 8th among 
China's 31 administrative regions, while Guangxi was the fifth-poorest province in the country (per capita GDP of USD 4939). ${ }^{15}$ Eligibility criteria for enrollment included a medical record indicating trabeculectomy surgery (concurrent cataract extraction was permitted, but not other ocular surgeries) and the ability to comply with the study protocol. After recruitment by study personnel and provision of informed consent, all patients were instructed orally by the operative surgeon to return for post-operative examinations at one and two weeks and one month after surgery. Trabeculectomy surgery was performed as an in-patient procedure at all participating facilities, and the initial post-operative examination was performed and questionnaires administered during this hospital stay, usually lasting 3 days. Pre-operative information, including presenting visual acuity in both eyes, and intra-ocular pressure and cup/disc ratio in the operative eye were abstracted from the inpatient chart if recorded.

Study questionnaires were administered by trained study personnel in the subject's local dialect of Chinese (Cantonese in Guangdong and Cantonese or Zhuang in Guangxi). Questions for patients concerned demographic and socioeconomic data, transportation status, knowledge and attitudes about glaucoma, glaucoma surgery and importance of follow-up, and the presence or absence of accompanying family members/friends for follow-up visits. Surgeons, in addition to providing demographic information, were asked about their work history, glaucoma surgical output, their own and perceive patient attitudes towards compliance, and whether they had instructed patients about the need to remove sutures post-operatively. Each interview took approximately half an hour to complete for patients and fifteen minutes for physicians. 
Data on subjects' compliance with follow up at one and two weeks, and one month after surgery were obtained from medical records. Follow-up was assessed by means of a score calculated as follows: follow-up at 1 week (3-11 days after discharge, 10 points), 2 weeks (12-21 days, 7 points) and 1 month (22-42 days, 5 points). Thus, the follow-up score ranged from 0 to 22, with the later denoting perfect follow-up. The rationale for weighting early follow-up more heavily was that interventions needing to be performed in this early post-operative period were likely to be more acute in nature, and thus impact more significantly on outcome. Similarly, reinforcing compliance with post-operative use of corticosteroids was felt to have a more significant impact on surgical outcome in the early post-operative period, when inflammation was expected to be most pronounced.

Results were presented as mean (SD, standard deviation) for data with normal distribution, otherwise, median (IQR, inter quarter range) and number (frequency) for categorical data. Patients' clinical and demographic characteristics, physician and hospital demographic and professional characteristics and other factors potentially associated with post-operative follow-up at both patient and physician levels were compared between Guangdong and Guangxi provinces. The two-sample t-test and Wilcoxon rank-sum test were used for continuous variables with and without normal distribution respectively, and Chi-square or Fisher's exact test were used for categorical data.

The main outcome, follow-up score, was divided into 8 ordered categories. Ordered logistic regression models were used to assess surgeon factors and patient age, sex, 
income, education, transportation situation, pre-operative presenting visual acuity, intraocular pressure and cup/disc ratio, awareness of and attitudes towards glaucoma and follow-up as potential predictors of follow-up score. Age, gender and all other variables significant at the $\mathrm{p}<0.05$ level in the simple regression were included in the multiple regression model. Robust 95\% confidence intervals were calculated.

Statistical analysis was done using a commercially available software package (Stata 12.0, StataCorp, College Station TX, USA).

\section{RESULTS}

Among 216 consecutive patients undergoing trabeculectomy at 14 rural county-level hospitals in Guangdong and Guangxi Provinces, 212 (98.1\%) enrolled and completed questionnaires. Their mean (SD) age was 60.2 (15.6) years, and 54.3\% $(115 / 212)$ were women. Nearly two-thirds of subjects $(135 / 212=63.7 \%)$ were engaged in farm work and 22.6\% (48/212) had had no formal education, while 39.6\% (84/212) had attended only elementary school (Table 1). Patients from Guangdong made up 48.6\% (103/212) of subjects and those from Guangxi 51.4\% (109/212). Patients from Guangxi were younger (57.5 (17.0) versus $63.0(13.5)$ years, $(\mathrm{P}=0.01)$, though the two groups did not differ significantly by gender, family income or the proportion working as farmers.

Roughly half of subjects from both Guangdong and Guangxi lived in rural villages, and proximity, travel time and travel modality to the local hospital did not differ significantly by province. However, patients in Guangxi were more likely to have the highest level of transportation costs $(22 / 109=20.2 \%$ versus $9 / 103=8.74 \%$ had costs $>$ 
USD 8, $\mathrm{P}=0.02)$, and to be without an accompanying friend or family member (44/109 $=40.4 \%$ versus $27 / 103=26.2 \%, \mathrm{P}=0.03)$.

Pre-operative clinical factors did not differ significantly by province, and glaucoma was severe in the operative eye in both settings. The mean (SD) LogMAR visual acuity overall was 1.19 (0.62) (approximately 6/120), with 61.3\% (n=130) being blind $(<=6 / 60)$ in the operative eye and $4.6 \%(n=6)$ blind in both eyes. The overall mean (SD) intra-ocular pressure in the operative eye was 38 (9.23) mmHg and median (IQR) cup-to-disc ratio (recorded for only 59 [27.8\%] of subjects) was 0.89 (0.80-1.00) (Table 2).

Table 3 gives information on subjects' knowledge and attitudes about glaucoma, glaucoma surgery and post-operative follow-up. Patients in Guangxi were more likely to believe that glaucoma would not lead to blindness $(40 / 109=36.7 \%$ versus $18 / 103=$ 17.4\% “Unlikely” or “Definitely not”, $\mathrm{P}=0.02$ ) and more commonly felt that glaucoma-induced blindness could “definitely” be cured (19/109 $=17.4 \%$ versus 5/103 $=4.85 \%, \mathrm{P}=0.04)$. Knowledge about how to treat and diagnose glaucoma was similar between the two groups, but patients in Guangxi were significantly less likely to know that follow-up after glaucoma surgery was "Very important” (14/109 = 12.8\% versus $43 / 103=41.8 \%, \mathrm{P}<0.001)$.

Table 4 summarizes physician and hospital factors potentially associated with patient compliance with follow-up. Professional and demographic factors were similar between the two provinces. All doctors in both settings agreed that post-operative follow-up was very important in determining results of glaucoma surgery, and the large 
majority $(12 / 14=85.7 \%)$ felt that post-operative compliance among their patients was only "Fair".

Over the initial post-operative month, 5.2\% of patients had no follow-up, 36.3\% of patients only attended a single follow-up visit and only $10.4 \%$ of subjects presented for all three follow-up visits. Post-operative follow-up rates were 60.8\% (129/212), 75.9\% (161/212) and 26.9\% (57/212) at 1 week, 2 weeks and 1 month respectively. Predictors of poor follow-up score in multiple regression models included having received no formal education $(\mathrm{OR}=0.37,95 \% \mathrm{CI}$ : $0.20,0.70, \mathrm{P}=0.002)$, believing follow-up was less important ( $\mathrm{OR}=0.62,95 \% \mathrm{CI}$ : 0.41, 0.94, $\mathrm{P}=0.02)$, lack of an accompanying friend or family member (OR $=0.14,95 \%$ CI: $0.07,0.29, \mathrm{P}<$ 0.001), annual family annual income $<$ USD 800 (OR $=0.28,95 \%$ CI: $0.11,0.72, \mathrm{P}=$ 0.008 ) and not requiring removal of a scleral flap suture post-operatively ( $\mathrm{OR}=0.11$, 95\% CI: 0.06, 0.22, P < 0.001). Age, sex, employment, transportation modality, distance, time and cost of travel, pre-operative patient clinical factors and physician factors were not significantly associated with follow-up (Table 5).The follow-up rates among patients with and without need for suture removal were as follows: 1 week: 82.6\% versus $42.1 \%(\mathrm{P}<0.001)$; 2 weeks: 93.9\% versus $60.5 \%(\mathrm{P}<0.001)$; and 1 month: $33.7 \%$ versus $21.1 \%(\mathrm{P}=0.04)$.

\section{DISCUSSION}

This is one of the first studies to examine pre-operative factors and compliance with recommended post-operative care after glaucoma surgery in rural areas of a lower or middle income country. The follow-up rates after trabeculectomy beyond 2 weeks in 
our study cohort (26.9\%) were not adequate to provide optimal care. Though high-quality trial evidence regarding the benefit of post-operative interventions on glaucoma outcomes may be lacking, the success and safety of glaucoma surgery is generally felt clinically to depend to an important extent on intensive postoperative management. . The American Academy of Ophthalmology's Preferred Practice Pattern for Open Angle Glaucoma, ${ }^{16}$ which has been adopted for use in China, recommends post-operative follow-up continue over at least 6 weeks, during which time the patient should be evaluated for complications, and a variety of treatments including injection of antifibrotic agents, repair of bleb leaks, bleb massage, suture lysis and bleb needling should be utilized as needed, in order to maximize the chances of long-term pressure control. Compared to cataract surgery, the outcome of glaucoma surgery is also more dependent on intensive use of topical corticosteroids during the pre-operative period, ${ }^{13}$ and it is likely that in this rural setting, few other sources would be available for such medications to patients not returning to clinic to receive them.

The fact that nearly two-thirds of these patients were already blind $(<=6 / 60)$ in the operative eye at the time of surgery suggests that treatment is being applied too late in these settings to reduce the burden of glaucoma blindness effectively. The possibility that some of these patients were blind wholly or in part due to cataract cannot be excluded. However, the mean IOP of 38 mmHg (with 76\% of IOP > 30 mmHg) suggests that this is less likely. While disc and field evaluation are normally required to diagnose glaucoma, and were not available for the majority of these 
patients, the International Society of Geographical and Epidemiological Ophthalmology (ISGEO) standards for the definition of glaucoma allow a diagnosis to be made in the event of blindness accompanied by an IOP exceeding the 99.5th percentile of the normal population. ${ }^{17}$ This would correspond in rural China to approximately $26.5 \mathrm{mmHg},{ }^{18}$ a figure exceeded by $91 \%$ of these patients. Furthermore, among the third of patients in whom cup to disc ratio was available, the mean was approximately 0.9. It seems likely that the majority of the blindness burden in these patients was due to glaucoma, and thus not treatable. While some of these patients were undergoing combined cataract extraction-trabeculectomy surgery, the median pre-operative visual acuity (inter-quartile range) in the operative eye was actually worse for patients undergoing trabeculectomy only (6/85 [6/150-6/30]) as compared to those with combined surgery (6/54 [6/120-6/30]). Earlier intervention appears to be needed, though it is possible that patients are only presenting for care, or only willing to accept surgery, when the vision has been severely affected. Further work is needed on the efficacy of interventions to promote more timely glaucoma surgery.

Guangdong and Guangxi had very different financial characteristics: the latter is one of China's poorest provinces and the former one of the richest. Thus, we sought to explore differences in follow-up and its determinants in these quite different settings. Patients in Guangxi reported higher transport costs and lower likelihood of having an accompanying person, and had lesser knowledge about glaucoma and in particular were less likely to know about the importance of follow-up. Though several of these factors were associated significantly with poor rates of follow-up in our models, it is 
interesting that mean follow-up in Guangxi did not differ from that in Guangdong. This suggests that there may be cultural or other local differences which we did not study and which prevented the expected worse compliance in Guangxi. This may in part be due to the fact that family income did not differ significantly between subjects by province. Perhaps those rural dwellers seeking surgery locally, rather than presenting to urban hospitals, represent less well-off populations in both Guangdong and Guangxi.

The relationship between physician communication and compliance has been highlighted by studies of patient acceptance of eye care services such as glaucoma medications ${ }^{19}$ and diabetic retinal examinations. ${ }^{20}$ Nonetheless, we failed to find any association between physician factors and patient adherence with recommended follow-up, other than the efficacy of recommending the patient return for suture removal. The current study may have included too few physicians to adequately assess the impact of physician factors. Further, many ophthalmologists in this cohort had similar attitudes about issues such as the importance of follow-up, which may not have been ideal for assessing the impact of different views. And finally, detailed aspects of physician-patient communication were not studied. The lack of significant association between patient pre-clinical factors and follow-up may be attributed in part to physicians not having communicated this information to patients, and also the fact that the majority of patients all had quite severe glaucoma at this juncture.

Few other studies exist on post-operative follow-up on ocular surgery in low and middle income countries, and the majority of these focus on cataract. One report on 
early assessment of cataract surgery at 40 hospitals in Africa, Asia and Latin America ${ }^{21}$ found that the 6 -week follow-up rate in China (27\%) was the lowest among 11 countries surveyed, while the mean follow-up rate across all institutions was 51\%. A report on post-cataract surgical follow-up from Africa $^{22}$ has detailed similar low rates of medium-term follow-up: $54 \%$ at 8 weeks post-operatively, though studies in rural China ${ }^{23,24}$ have demonstrated that phone contact and offers of compensation can result in follow-up at 3-12 months in excess of $80 \%$. The very few studies reporting follow-up rates after glaucoma surgery in developing areas give somewhat higher figures: $84 \%$ at 1 month in China ${ }^{14}$ and $58 \%$ at a year in India, ${ }^{9}$ though neither study was designed specifically to assess follow-up under routine conditions, and these results may not be representative. Among the few studies reporting on determinants of post-operative follow-up after eye surgery in low and middle income countries, lack of knowledge, ${ }^{14}$ rural residence, ${ }^{14}$ visual impairment, ${ }^{14}$ low income ${ }^{23}$ and failure to recall physician’s instructions to return ${ }^{24}$ were risk factors for poor compliance.

Determinants of better adherence with recommended post-operative follow-up in the current study do suggest potential strategies to improve patient rates of return. The first is educational interventions: lack of importance attached to follow-up visits was a significant risk factor for poor follow-up, an issue which might readily be addressed through patient instruction or viewing of videos. Our previous work in southern rural China, however, has shown that educational interventions were not sufficient in and of themselves to promote purchase of spectacles, ${ }^{25}$ or acceptance of cataract surgery ${ }^{26}$ or comprehensive eye examinations. ${ }^{27}$ However, our finding that low income and lack 
of an accompanying person were important risk factors for failure to return to clinic as directed suggests that financial incentives to patients, family members and other potential escorts could also be of value, and might be combined with educational interventions. Financial incentives might include free medications or cellphone top-ups, as previous focus group studies have indicated that patients in rural China are uncomfortable with direct cash payments to encourage medical compliance. ${ }^{28}$ Shortening waiting times in the clinic might also decrease escorts' burden of time spent and wages lost. More innovative strategies might include employing a hospital-run escort and/or transportation system, organizing a community-based escort pool by which a single person could accompany multiple patients from the community, or providing follow-up care locally at existing community health centers. Each of these would require substantial investment, presumably from the government, to be sustainable.

Although the project protocol required physicians to inform patients of the need to return to clinic three times in the first month, follow-up rates across the three visits among patients requiring scleral flap suture removal were $50-100 \%$ greater than for those not requiring such care. Simply telling all patients that they should be certain to return for each of the three visits in view of the possible need to remove scleral flap sutures may be a simple, inexpensive and effective strategy to improve post-operative adherence in this setting.

Strengths of the current study include having enrolled a comparatively large number of hospitals in two financially very different settings, the prospective design 
ensuring collection of data required by the study protocol, the selection of a rural setting where surgery is the primary treatment for glaucoma, and the fact that adherence with post-operative care has been demonstrated to be an important determinant of glaucoma surgical outcomes. ${ }^{11-13}$ Weaknesses must also be acknowledged: application of these results beyond these institutions and geographic settings may be made only with care, and it is possible that participation in a study which queried patients about the importance of follow-up might have influenced their behavior. Moreover, the current study did not attempt to link post-operative adherence with surgical outcomes; a future report will attempt to address this issue with a larger cohort. Gonioscopy was rarely performed and recorded in these settings, and so we are unable to state whether these cases represented open-angle or angle-closure glaucoma. Despite this limitation, given the relatively large number of hospitals involved, 14 across two provinces, it is likely that this series given an indication of the case-mix at rural hospitals in southern China, which was the goal of the current study, rather than to assess the relative prevalence of glaucoma sub-types. Finally, the study was not well-designed to assess the impact of potentially important physician factors, in that the number of physician subjects was small and in-depth interviews and observation of patient encounters were not carried out.

Despite its limitations, this study provides some of the first data on pre-operative status and follow-up after glaucoma surgery in a rural low-middle income country setting. It is hoped that these results may help to inform interventions to increase current low follow-up rates and promote earlier surgery as part of improving surgical 
outcomes. 
Table 1. Demographic characteristics of patients undergoing trabeculectomy surgery recruited in Guangdong and Guangxi provinces

\begin{tabular}{|c|c|c|c|c|}
\hline & $\begin{array}{l}\text { All } \\
(\mathrm{N}=212)\end{array}$ & $\begin{array}{l}\text { Guangdong } \\
(N=103,48.6 \%)\end{array}$ & $\begin{array}{l}\text { Guangxi } \\
(N=109,51.4 \%)\end{array}$ & $P$ Value $^{\mathrm{a}}$ \\
\hline Age, years, Mean(SD) & $60.2(15.6)$ & $63.0(13.5)$ & $57.5(17.0)$ & 0.01 \\
\hline Female, No. (\%) & $115(54.3)$ & $62(60.2)$ & $53(48.6)$ & 0.09 \\
\hline \multicolumn{5}{|l|}{ Employment, No.(\%) } \\
\hline Working in agriculture & $135(63.7)$ & $60(58.2)$ & $75(68.8)$ & \multirow{2}{*}{0.11} \\
\hline Other & $77(36.3)$ & $43(41.8)$ & $34(31.2)$ & \\
\hline \multicolumn{5}{|l|}{ Education, No. (\%) } \\
\hline No schooling or elementary school & $132(62.3)$ & $65(63.1)$ & $67(61.5)$ & \multirow{2}{*}{0.81} \\
\hline Higher than elementary school & $80(37.7)$ & $38(36.9)$ & $42(38.5)$ & \\
\hline \multicolumn{5}{|l|}{ Marital status, No. (\%) } \\
\hline Married & $160(75.5)$ & $81(78.6)$ & 79 (72.5) & \multirow{2}{*}{0.30} \\
\hline Divorce / Widowed / Unmarried & $52(24.5)$ & $22(21.4)$ & $30(27.5)$ & \\
\hline \multicolumn{5}{|l|}{ Family annual income, US\$, No.(\%) } \\
\hline$<\$ 800$ & $45(21.2)$ & $24(23.3)$ & $21(19.3)$ & \multirow{4}{*}{0.17} \\
\hline$\$ 800-1,600$ & $67(31.6)$ & $26(25.2)$ & $41(37.6)$ & \\
\hline$\$ 1,600-8,000$ & 78 (36.8) & $39(37.9)$ & $39(35.8)$ & \\
\hline$>\$ 8,000$ & $22(10.4)$ & $14(13.6)$ & $8(7.34)$ & \\
\hline
\end{tabular}

Abbreviations: SD, standard deviation

a The two-sample t test was used for age and the Chi-square test for comparisons between the two provinces. 
Table 2. Factors potentially associated with post-operative follow-up among patients undergoing trabeculectomy in Guangdong and Guangxi provinces

\begin{tabular}{|c|c|c|c|c|}
\hline & $\begin{array}{l}\text { All } \\
(\mathrm{N}=212)\end{array}$ & $\begin{array}{l}\text { Guangdong } \\
(\mathrm{N}=103)\end{array}$ & $\begin{array}{l}\text { Guangxi } \\
(\mathrm{N}=109)\end{array}$ & $P$ Value $^{\mathrm{a}}$ \\
\hline Rural (Village / Town), No. (\%) & $164(77.4)$ & $81(78.6)$ & $83(76.2)$ & 0.67 \\
\hline \multicolumn{5}{|c|}{$\begin{array}{l}\text { Distance from home to hospital, No. } \\
(\%), \mathrm{km}\end{array}$} \\
\hline$<10$ & $85(40.1)$ & $40(38.8)$ & $45(41.3)$ & \multirow{2}{*}{0.72} \\
\hline$>=10$ & 127 (59.9) & $63(61.2)$ & $64(58.7)$ & \\
\hline
\end{tabular}

Travel time from home to hospital, No.

(\%), hours

\begin{tabular}{|c|c|c|c|c|}
\hline$<1$ & 149 (70.3) & $78(75.7)$ & $71(65.1)$ & \multirow{2}{*}{0.09} \\
\hline$>=1$ & $63(29.7)$ & $25(24.3)$ & $38(34.9)$ & \\
\hline \multicolumn{5}{|l|}{ Transportation, No. (\%) } \\
\hline By public transit & $99(46.7)$ & $46(44.7)$ & $53(48.6)$ & \multirow[b]{2}{*}{0.56} \\
\hline $\begin{array}{l}\text { By other modes (private car / motorcycle } \\
\text { / bicycle / on foot) }\end{array}$ & $113(53.3)$ & $57(55.3)$ & $56(51.4)$ & \\
\hline \multicolumn{5}{|l|}{ Convenience of transportation, No. (\%) } \\
\hline Convenient & $33(15.6)$ & $15(14.6)$ & $18(16.5)$ & \multirow{5}{*}{0.32} \\
\hline Very convenient & $76(35.9)$ & $43(41.8)$ & $33(30.3)$ & \\
\hline Somewhat inconvenient & $55(25.9)$ & $24(23.3)$ & $31(28.4)$ & \\
\hline Inconvenient & $40(18.9)$ & $19(18.5)$ & $21(19.3)$ & \\
\hline Very inconvenient & $8(3.77)$ & $2(1.94)$ & $6(5.50)$ & \\
\hline \multicolumn{5}{|l|}{ Transportation cost, No. (\%), US\$ } \\
\hline$<=\$ 8$ & $181(85.4)$ & $94(91.3)$ & $87(79.8)$ & \multirow{2}{*}{0.02} \\
\hline$>\$ 8$ & $31(14.6)$ & $9(8.74)$ & $22(20.2)$ & \\
\hline
\end{tabular}

Accompanying friend or family

member, No. (\%)

\begin{tabular}{|c|c|c|c|c|}
\hline Yes & $141(66.5)$ & $76(73.8)$ & $65(59.6)$ & \multirow{2}{*}{0.03} \\
\hline No & $71(33.5)$ & $27(26.2)$ & $44(40.4)$ & \\
\hline \multicolumn{5}{|l|}{ Pre-operative presenting visual acuity } \\
\hline \multicolumn{5}{|l|}{ in operated eye, mean (SD), LogMAR } \\
\hline LogMAR notation & $1.19(0.62)$ & $1.20(0.62)$ & $1.19(0.62)$ & $0.97^{b}$ \\
\hline Snellen equivalent & $20 / 310$ & $20 / 320$ & $20 / 310$ & \\
\hline $\begin{array}{l}\text { Pre-operative intraocular pressure, } \\
\text { mean (SD) , mmHg }\end{array}$ & $38.0(9.23)$ & $38.6(10.1)$ & $37.5(8.36)$ & $0.39^{b}$ \\
\hline Pre-operative Cup/Disc ratio, median & 0.90 & 0.90 & 0.90 & $050 \mathrm{~d}$ \\
\hline$(\mathrm{IQR})^{\mathrm{c}}$ & $(0.80-1.00)$ & $(0.80-1.00)$ & $(0.80-1.00)$ & 0.02 \\
\hline Need to remove suture & & & & 0.92 \\
\hline \multicolumn{5}{|l|}{ post-operatively, No. (\%) } \\
\hline Yes & $98(46.2)$ & $48(46.6)$ & $50(45.9)$ & \\
\hline No & $114(53.8)$ & $55(53.4)$ & $59(54.1)$ & \\
\hline
\end{tabular}

Abbreviations: SD, standard deviation; LogMAR, log of the minimum angle of resolution; IQR, Inter quartile range. 
${ }^{a}$ Chi-square test unless otherwise noted for comparisons between the two provinces.

b Two-sample t test

c $153(153 / 212,72.2 \%)$ of subjects had missing data on Cup/Disc ratio.

d Wilcoxon rank-sum test 
Table 3.Patient knowledge and attitudes about glaucoma surgery and follow-up

\begin{tabular}{|c|c|c|c|c|}
\hline & $\begin{array}{l}\text { All } \\
(\mathrm{N}=212)\end{array}$ & $\begin{array}{l}\text { Guangdong } \\
(\mathrm{N}=103)\end{array}$ & $\begin{array}{l}\text { Guangxi } \\
\text { (N=109) }\end{array}$ & $P$ Value $^{\mathrm{a}}$ \\
\hline \multicolumn{5}{|l|}{$\begin{array}{l}\text { Glaucoma can lead to blindness, No. } \\
\text { (\%) }\end{array}$} \\
\hline Definitely & $51(24.1)$ & $30(29.1)$ & $21(19.3)$ & \multirow{4}{*}{0.02} \\
\hline Maybe & $103(48.6)$ & $55(53.4)$ & $48(44.0)$ & \\
\hline Unlikely & $51(24.1)$ & $16(15.5)$ & $35(32.1)$ & \\
\hline Definitely not & $7(3.30)$ & $2(1.94)$ & $5(4.59)$ & \\
\hline \multicolumn{5}{|l|}{$\begin{array}{l}\text { Glaucoma-induced blindness can be } \\
\text { cured, No. (\%) }\end{array}$} \\
\hline Definitely & $24(11.3)$ & $5(4.85)$ & $19(17.4)$ & \multirow{4}{*}{0.04} \\
\hline Maybe & $101(47.6)$ & $52(50.5)$ & $49(45.0)$ & \\
\hline Unlikely & $71(33.5)$ & $38(36.9)$ & $33(30.3)$ & \\
\hline Definitely not & $16(7.55)$ & $8(7.77)$ & $8(7.34)$ & \\
\hline $\begin{array}{l}\text { Knows glaucoma can be treated } \\
\text { surgically, No. (\%) }\end{array}$ & $165(77.8)$ & $86(83.5)$ & $79(72.5)$ & 0.05 \\
\hline $\begin{array}{l}\text { Knows glaucoma can be diagnosed in } \\
\text { the early phase, No. (\%) }\end{array}$ & $189(89.1)$ & $94(91.3)$ & $95(87.2)$ & 0.34 \\
\hline \multicolumn{5}{|l|}{$\begin{array}{l}\text { Importance of follow-up after surgery, } \\
\text { No. (\%) }\end{array}$} \\
\hline Very important & $57(26.9)$ & $43(41.8)$ & $14(12.8)$ & \multirow{5}{*}{$<0.001$} \\
\hline Important & $76(35.9)$ & $30(29.1)$ & $46(42.2)$ & \\
\hline Somewhat important & $67(31.6)$ & $28(27.2)$ & $39(35.8)$ & \\
\hline Not important & $12(5.66)$ & $2(1.94)$ & $10(9.17)$ & \\
\hline Not important at all & $0(0.00)$ & $0(0.00)$ & $0(0.00)$ & \\
\hline
\end{tabular}

a Chi-square test was used for comparisons between the two provinces. 
Table 4. Physician and hospital factors potentially associated with patient compliance with post-operative follow up in Guangdong and Guangxi

\begin{tabular}{|c|c|c|c|c|}
\hline & $\begin{array}{l}\text { All } \\
(\mathrm{N}=14)\end{array}$ & $\begin{array}{l}\text { Guangdong } \\
(\mathrm{N}=7)\end{array}$ & $\begin{array}{l}\text { Guangxi } \\
(N=7)\end{array}$ & $P$ Value \\
\hline \multicolumn{5}{|c|}{$\begin{array}{l}\text { Demographic and Professional } \\
\text { Characteristics }\end{array}$} \\
\hline \multicolumn{5}{|l|}{ Age, year, No. (\%) } \\
\hline $30-40$ & $5(35.7)$ & $1(14.3)$ & $4(57.1)$ & \multirow{3}{*}{0.34} \\
\hline $41-50$ & $9(64.3)$ & $6(85.7)$ & $3(42.9)$ & \\
\hline Mean (SD) & $40.6(4.45)$ & $41.9(4.74)$ & $39.4(4.11)$ & \\
\hline Male, No. (\%) & $10(71.4)$ & $7(100.0)$ & $3(42.9)$ & 0.07 \\
\hline \multicolumn{5}{|c|}{ Hospital characteristics, No. (\%) } \\
\hline Special hospital & $1(7.14)$ & $1(14.3)$ & $0(0.00)$ & \multirow{2}{*}{1.00} \\
\hline General hospital & $13(92.9)$ & $6(85.7)$ & $7(100.0)$ & \\
\hline \multicolumn{5}{|c|}{ Professional title, No. (\%) } \\
\hline Attending doctor & $12(85.7)$ & $6(85.7)$ & $6(85.7)$ & \multirow{2}{*}{1.00} \\
\hline Resident doctor & $2(14.3)$ & $1(14.3)$ & $1(14.3)$ & \\
\hline \multicolumn{5}{|c|}{$\begin{array}{l}\text { Time of working in ophthalmology, } \\
\text { years, No. (\%) }\end{array}$} \\
\hline$<=10$ & $3(21.4)$ & $1(14.3)$ & $2(28.6)$ & \multirow{2}{*}{1.00} \\
\hline$>10$ & $11(78.6)$ & $6(85.7)$ & $5(71.4)$ & \\
\hline
\end{tabular}

Annual volume of glaucoma

surgeries, No. (\%)

\begin{tabular}{|c|c|c|c|c|}
\hline$<=20$ & $8(57.2)$ & $3(42.8)$ & 5 (71.4) & \multirow{4}{*}{0.63} \\
\hline $21-40$ & $3(21.4)$ & $2(28.6)$ & $1(14.3)$ & \\
\hline$>40$ & $3(21.4)$ & $2(28.6)$ & $1(14.3)$ & \\
\hline Mean (SD) & $29.3(14.8)$ & $34.3(16.2)$ & $24.3(12.4)$ & \\
\hline \multicolumn{5}{|c|}{ Physician Knowledge and Attitudes } \\
\hline \multicolumn{5}{|c|}{$\begin{array}{l}\text { Importance of follow-up after } \\
\text { surgery at } 1 \text { month, No. (\%) }\end{array}$} \\
\hline Very important & $14(100.0)$ & $7(100.0)$ & $7(100.0)$ & $\ldots$ \\
\hline \multicolumn{5}{|c|}{$\begin{array}{l}\text { How is compliance with } \\
\text { post-operative follow-up among } \\
\text { your patients, No. (\%) }\end{array}$} \\
\hline Very good & $0(0.00)$ & $0(0.00)$ & $0(0.00)$ & \multirow{5}{*}{0.46} \\
\hline Good & $2(14.3)$ & $0(0.00)$ & $2(28.6)$ & \\
\hline Fair & $12(85.7)$ & $7(100.0)$ & $5(71.4)$ & \\
\hline Poor & $0(0.00)$ & $0(0.00)$ & $0(0.00)$ & \\
\hline Very poor & $0(0.00)$ & $0(0.00)$ & $0(0.00)$ & \\
\hline
\end{tabular}

Do you see your post-operative

patients personally, No. (\%)

\begin{tabular}{lllll} 
Always see & $1(7.14)$ & $1(14.3)$ & $0(0.00)$ & \multirow{2}{*}{1.00} \\
Usually see & $12(85.7)$ & $6(85.7)$ & $6(85.7)$ & \\
\hline
\end{tabular}


a Two sample t-test was used for age and annual volume of glaucoma surgeries, and Fisher's exact test was used for other variables for comparisons between the two provinces. 
Table 5: Ordered logistic regression of potential determinants of follow-up score ${ }^{a}$ (items significant at the $P<0.05$ level are highlighted in bold)

\begin{tabular}{|c|c|c|c|c|}
\hline \multirow{3}{*}{ Variable } & \multicolumn{2}{|c|}{ Simple model } & \multicolumn{2}{|c|}{ Multiple model ${ }^{b}$} \\
\hline & Odds ratio & & Odds ratio & \\
\hline & $(95 \% \mathrm{Cl})$ & $P$ Value & $(95 \% \mathrm{Cl})$ & $P$ Value \\
\hline \multicolumn{5}{|l|}{ Patient level } \\
\hline Age, per year & $1.00(0.99,1.02)$ & 0.81 & $1.00(0.98,1.02)$ & 0.96 \\
\hline Female & $1.19(0.73,1.94)$ & 0.49 & $1.33(0.75,2.34)$ & 0.33 \\
\hline Working in agriculture & $0.85(0.53,1.38)$ & 0.52 & & \\
\hline $\begin{array}{l}\text { No schooling or elementary } \\
\text { school }\end{array}$ & $0.17(0.09,0.30)$ & $<0.001$ & $0.37(0.20,0.70)$ & 0.002 \\
\hline Married & $1.28(0.72,2.28)$ & 0.40 & & \\
\hline \multicolumn{5}{|l|}{ Family annual income, US\$ } \\
\hline$<806.5$ & $0.17(0.06,0.49)$ & 0.001 & $0.28(0.11,0.72)$ & 0.008 \\
\hline $806.5-1,612.9$ & $0.33(0.13,0.84)$ & 0.02 & $0.63(0.30,1.34)$ & 0.23 \\
\hline $1,612.9-8,064.5$ & $0.34(0.13,0.90)$ & 0.03 & $0.38(0.17,0.84)$ & 0.02 \\
\hline$>8,064.5$ & Reference & & Reference & \\
\hline $\begin{array}{l}\text { Importance of follow-up after } \\
\text { surgery }{ }^{c}\end{array}$ & $0.31(0.22,0.46)$ & $<0.001$ & $0.62(0.41,0.94)$ & 0.02 \\
\hline $\begin{array}{l}\text { Glaucoma can lead to } \\
\text { blindness }^{c}\end{array}$ & $0.93(0.67,1.28)$ & 0.64 & & \\
\hline $\begin{array}{l}\text { Glaucoma-induced blindness } \\
\text { can be cured }\end{array}$ & $1.39(1.00,1.92)$ & 0.05 & & \\
\hline $\begin{array}{l}\text { Knows glaucoma can be } \\
\text { treated surgically }\end{array}$ & $0.80(0.49,1.30)$ & 0.37 & & \\
\hline $\begin{array}{l}\text { Knows glaucoma can be } \\
\text { diagnosed in early phase }\end{array}$ & $1.34(0.56,3.21)$ & 0.51 & & \\
\hline $\begin{array}{l}\text { Rural (Village/town) versus } \\
\text { urban residence }\end{array}$ & $1.46(0.76,2.78)$ & 0.26 & & \\
\hline Lives $>=10 \mathrm{~km}$ from hospital & $0.95(0.58,1.54)$ & 0.83 & & \\
\hline Lives $>=1$ hour from hospital & $0.57(0.33,0.99)$ & 0.045 & $0.82(0.41,1.64)$ & 0.57 \\
\hline $\begin{array}{l}\text { Public transit versus other } \\
\text { ways }\end{array}$ & $0.91(0.55,1.50)$ & 0.72 & & \\
\hline $\begin{array}{l}\text { Convenience of } \\
\text { transportation }^{c}\end{array}$ & $0.97(0.76,1.24)$ & 0.80 & & \\
\hline Transportation fees>US\$8 & $1.09(0.63,1.89)$ & 0.75 & & \\
\hline Has no accompanying friend & $0.08(0.05,0.14)$ & $<0.001$ & $0.14(0.07,0.29)$ & $<0.001$ \\
\hline
\end{tabular}




\begin{tabular}{|c|c|c|c|c|}
\hline \multicolumn{5}{|l|}{ or family member } \\
\hline $\begin{array}{l}\text { Pre-operative presenting } \\
\text { visual acuity, LogMAR }\end{array}$ & $1.27(0.87,1.87)$ & 0.21 & & \\
\hline $\begin{array}{l}\text { Pre-operative intraocular } \\
\text { pressure, } \mathrm{mmHg}\end{array}$ & $1.01(0.99,1.04)$ & 0.39 & & \\
\hline Pre-operative Cup/Disc ratio & $1.13(0.01,99.2)$ & 0.96 & & \\
\hline No need for suture removal & $0.05(0.03,0.10)$ & $<0.001$ & $0.11(0.06,0.22)$ & $<0.001$ \\
\hline $\begin{array}{l}\text { Province (Guangxi as } \\
\text { reference) }\end{array}$ & $1.10(0.68,1.78)$ & 0.70 & $0.56(0.30,1.06)$ & 0.07 \\
\hline \multicolumn{5}{|l|}{ Physician level } \\
\hline Age, year & $0.98(0.92,1.04)$ & 0.46 & & \\
\hline Female & $0.83(0.50,1.40)$ & 0.50 & & \\
\hline $\begin{array}{l}\text { Attending doctor versus } \\
\text { resident doctor }\end{array}$ & $0.48(0.22,1.07)$ & 0.07 & & \\
\hline $\begin{array}{l}>10 \text { years working in } \\
\text { ophthalmology }\end{array}$ & $0.79(0.40,1.57)$ & 0.50 & & \\
\hline \multicolumn{5}{|l|}{$\begin{array}{l}\text { Annual volume of glaucoma } \\
\text { surgery }\end{array}$} \\
\hline$<=20$ & Reference & & & \\
\hline $21-40$ & $0.67(0.35,1.28)$ & 0.23 & & \\
\hline$>40$ & $0.75(0.43,1.28)$ & 0.29 & & \\
\hline $\begin{array}{l}\text { Perceived compliance with } \\
\text { follow-up after surgery among } \\
\text { patients }^{c}\end{array}$ & $1.27(0.75,2.15)$ & 0.37 & & \\
\hline
\end{tabular}

Abbreviations: $\mathrm{Cl}$, confidence interval; LogMAR, log of the Minimum Angle of Resolution.

${ }^{\text {a }}$ Follow-up score is the sum of scores assigned for patients followed up at 1 week: 1 11 days (10 points), 2 week: 12 21 days (7 points) and 1 month: 22 42 days (5 points).

${ }^{\mathrm{b}}$ Age, gender and all other variables with $p<0.05$ in the simple regression were included in the multiple regression.

${ }^{c}$ The variable was defined on a 5-stage Likert scale and treated as continuous. 
Contributors NC contributed in the form of designing,data analysis,manuscript preparation and submission. KY contributed to the collection of data,analysis of results and drafting of manuscript. LJ contributed to the data analysis.

LL,SMZ,AHD,TTC,XQW,GRL contributed to the collection of data.

Funding Financial support provided by Orbis International, Zhongshan Ophthalmic Center and the World Diabetes Foundation. Prof Congdon is supported by the Chinese government Thousand Man Plan and by the Ulverscroft Foundation.

Competing interests None.

Ethics approval Zhongshan Ophthalmic Center Ethics Committee, Sun Yat-sen University.

Provenance and peer review Not commissioned;externally peer reviewed. 


\section{REFERENCES}

1. Tham YC, Li X, Wong TY, et al. Global Prevalence of Glaucoma and Projections of Glaucoma Burden through 2040. Ophthalmology.2014; 121(11):2081-2090.

2. Xu L, Wang Y, Li Y, et al. Causes of blindness and visual impairment in urban and rural areas in Beijing: the Beijing Eye Study. Ophthalmology.2006; 113(7):1134.e1-11.

3. Liang YB, Friedman DS, Wong TY, et al. Prevalence and causes of low vision and blindness in a rural Chinese adult population: the Handan Eye Study. Ophthalmology.2008; 115(11):1965-1972.

4. Huang S, Zheng Y, Foster PJ, et al. Prevalence and Causes of Visual Impairment in Chinese Adults in Urban Southern China. Arch Ophthalmol.2009; 127(10):1362-1367.

5. Leske MC, Heijl A, Hussein M, et al. Factors for glaucoma progression and the effect of treatment: the Early Manifest Glaucoma Trial. Arch Ophthalmol.2003; 121 (1):48-56.

6. Heijl A, Leske MC, Bengtsson B, et al. Reduction of intraocular pressure and glaucoma progression: results from the Early Manifest Glaucoma Trial. Arch Ophthalmol.2002; 120(10):1268-1279.

7. Lichter PR, Musch DC, Gillespie BW, et al. Interim clinical outcomes in the Collaborative Initial Glaucoma Treatment Study comparing initial treatment randomized to medications or surgery. Ophthalmology.2001; 108(11):1943-1953.

8. Nayak B, Gupta S, Kumar G, et al. Socioeconomics of long-term glaucoma 
therapy in India. Indian Journal of Ophthalmology.2015; 63(1):20-24.

9. Congdon NG, Krishnadas R, Friedman DS, et al. A Study of Initial Therapy for Glaucoma in Southern India: India Glaucoma Outcomes and Treatment (INGOT) Study. Ophthalmic Epidemiology.2012; 19(3):149-158.

10. Liang Y, Wang N, Rong S, et al. Initial Treatment for Primary Angle-Closure Glaucoma in China. Journal of Glaucoma.2014; 24(6):469-473.

11. Singh J, Bell RW, Adams A, et al. Enhancement of post trabeculectomy bleb formation by laser suture lysis. Br J Ophthalmol.1996; 80(7):624-627.

12. Henderer JD, Heeg MC, Spaeth GL, et al. A Randomized Trial of the Long-term Effects of Digital Ocular Compression in the Late Postoperative Period. $J$ Glaucoma.2001; 10(4):266-270.

13. Roth SM, Spaeth GL, Starita RJ, et al. The effects of postoperative corticosteroids on trabeculectomy and the clinical course of glaucoma: five-year follow-up study. Ophthalmic Surg.1991; 22(12):724-729.

14. Liu K, Rong S, Liang Y, et al. A retrospective survey of long-term follow-up on primary angle closure glaucoma after trabeculectomy. Ophthalmol CHN.2011; 20(1):50-53.

15. Wikipedia: list of Chinese administrative divisions by GDP. Available at: http://en.wikipedia.org/

wiki /List_of_Chinese_administrative_divisions_by_GDP_per_capita. Last accessed March 2, 2015.

16. The American Academy of Ophthalmology Preferred Practice Patterns 
Committee. Glaucoma Panel. Preferred Practice Patterns: Primary Open-angle Glaucoma.http://www.aao.org/preferred-practice-pattern/primary-openangle-glaucom a-ppp--october-2010. Website last accessed 12 June 2015.

17. Foster PJ, Buhrmann R, Quigley HA, et al. The definition and classification of glaucoma in prevalence surveys. Br J Ophthalmol. 2002;86(2):238-242.

18. Xu L, Li J, Zheng Y, et al. Intraocular Pressure in Northern China in an Urban and Rural Population: The Beijing Eye Study. Am J Ophthalmol.2005;140:913-915.

19. Friedman DS, Hahn SR, Gelb L, et al. Doctor-Patient Communication, Health-Related Beliefs, and Adherence in Glaucoma.Ophthalmology.2008; 115(8):1320-1327.

20. Wang D, Ding X, He M, et al. Use of Eye Care Services among Diabetic Patients in Urban and Rural China. Ophthalmology.2010; 117(9):1755-1762.

21. Congdon N, Yan X, Lansingh V, et al. Assessment of cataract surgical outcomes in settings where follow-up is poor: PRECOG, a multicentre observational study. Lancet Glob Health.2013; 1(1):e37-45.

22. Limburg H, Foster A, Gilbert C, et al. Routine monitoring of visual outcome of cataract surgery. Part 2: Results from eight study centre’s. Br J Ophthalmol.2005; 89(1):50-52.

23. Huang G, Crooms R, Chen Q, et al. Compliance with Follow-up after Cataract Surgery in Rural China. Ophthalmic Epidemiology.2012; 19 (2):67-73.

24. Lam DS, Congdon NG, Rao SK, et al. Visual Outcomes and Astigmatism after Sutureless, Manual Cataract Extraction in Rural China. Arch Ophthalmol. 2007; 
125(11):1539-1544

25. Congdon N, Li L, Zhang M, et al. Randomized, controlled trial of an educational intervention to promote spectacle use in rural China: the see well to learn well study. Ophthalmology.2011; 118 (12):2342-2350.

26. Liu T, Congdon N, Yan X, et al. A Randomized, Controlled Trial of an Intervention Promoting Cataract Surgery Acceptance in Rural China: The Guangzhou Uptake of Surgery Trial (GUSTO). Invest Ophthalmol Vis Sci.2012; 53 (9):5271-5278.

27. Dan A, Raubvogel G, Chen T, et al. The Impact of Multimedia Education on Uptake of Comprehensive Eye Examinations in Rural China: A Randomized, Controlled Trial. Ophthalmic Epi. 2015; 22(4):283-290.

28. Yan X, Liu T, Gruber L, et al. Attitudes of Physicians, Patients, and Village Health Workers Toward Glaucoma and Diabetic Retinopathy in Rural China. Arch Ophthalmol. 2012; 130(6):761-770. 